Military Technical College

Kobry Elkobbah,

Cairo, Egypt

April 19-21,2016

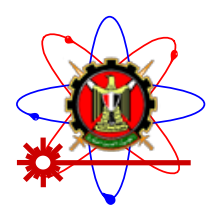

$8^{\text {th }}$ International Conference on Mathematics and Engineering

Physics (ICMEP-8)

\title{
EM-9
}

\section{Dynamic Stress Intensity Factors for an Interfacial Crack near a Non-Circular Cavity in Piezoelectric Bi-Materials}

\begin{abstract}
Ahmed Hassan
MTC

Abstract

A theoretical analysis is followed to calculate the dynamic stress intensity factors (DSIFs) due to existence of an interfacial crack near the right edge of a non-circular cavity, in transversely isotropic piezoelectric bi-materials. The model is subjected to dynamic incident anti-plane shearing (SH-wave). Green's functions are constructed Based on complex variable and conformal mapping methods. DSIFs at the crack inner and outer tips are obtained by conjunction and cracks-deviation techniques. The boundary value problems are solved by applying the orthogonal function expansion technique. Based on FORTRAN language program, some numerical calculations with an elliptic cavity are provided for different elliptic axial length ratios, different wave numbers and different piezoelectric parameters. For calibration, a comparison is accomplished between the present model and similar model with a crack emerging from a circular cavity edge. Calculating results showed the influences on DSIFs and how affected the efficiency of piezoelectric devices and materials.
\end{abstract}

